\title{
Episodic retrieval and the cortical binding of relational activity
}

\author{
Arthur P. Shimamura
}

Published online: 3 June 2011

(C) Psychonomic Society, Inc. 2011

\begin{abstract}
Retrieval of episodic memories depends on the successful "re-collection" of event features, such as the time, place, people, thoughts, and feelings associated with a past experience.In neuroimaging studies, ventral regions of the posterior parietal cortex (vPPC) are particularly active when episodic memories are successfully retrieved. A review of the neural correlates of episodic retrieval is presented along with a new theory, cortical binding of relational activity (CoBRA). According to CoBRA, the vPPC acts as a convergence zone that binds episodic features stored in disparate neocortical regions. This process works in conjunction with other known mechanisms, such as those associated with the prefrontal cortex and medial temporal lobe.
\end{abstract}

Keywords Episodic memory $\cdot$ Parietal cortex $\cdot$ Recollection

What did you do last weekend? Our ability to retrieve such episodic memories depends on efficient storage of event features at the time of encoding. These features include the sensory, conceptual, and emotional experiences that define an event. During encoding, event features distributed across neocortical sites are held online by the prefrontal cortex (PFC) as an active working memory representation (D'Esposito, 2007; Miller \& Cohen, 2001; Shimamura, 2000, 2008). The medial temporal lobe (MTL) binds these cortical representations, thus enabling retrieval of them at a

This research was supported by NSF Grant BCS-0745835.

A. P. Shimamura $(\bowtie)$

Department of Psychology (MC1650), University of California,

Berkeley, CA 94720-1650, USA

e-mail: aps@berkeley.edu later time (Cohen \& Eichenbaum, 1993; Shimamura, 2010; Shimamura and Wickens 2009; Squire \& Zola, 1998). Considerable neuroimaging research has shown that PFC and MTL activity during encoding is strongly correlated with subsequent memory retrieval (see Paller \& Wagner, 2002).

A prominent feature of episodic retrieval is the reinstatement of source memory, which refers to memory for the contextual features that make up a prior event (Johnson, Hashtroudi, \& Lindsay, 1993; Mitchell \& Johnson, 2009). Source memory includes information about the time, place, people, sights, thoughts, and feelings that define an episodic memory. Tests of source memory assess the ability to retrieve such information by asking individuals to recollect specific contextual features, such as the color or location of a previously presented stimulus. Source memory has been considered an essential, if not defining, characteristic of episodic memory (Shimamura \& Wickens, 2009; Tulving, 2002; Yonelinas, 2002). Indeed, successful episodic retrieval is determined largely by the number of source memory features that can be reinstated. Other terms, such as contextual memory and relational memory, capture the importance of binding source memory as an encapsulated ensemble of event features.

Figure 1 outlines critical components involved in the encoding of episodic memory. Initially, bottom-up sensory processes, such as visual processing along the dorsal and ventral paths, are activated in response to sensory signals. Top-down PFC processes act to select, maintain, and update relevant event features, which comprise the multitude of conceptual, emotional, and sensory features that make up an episodic event. On the basis of consolidation theory (Cohen \& Eichenbaum, 1993; Shimamura, 2002; Squire, Shimamura, \& Amaral, 1989; Squire \& Zola, 1998), theMTL binds coactive feature sites stored in disparate regions in the neocortex. Binding is established by way of a 


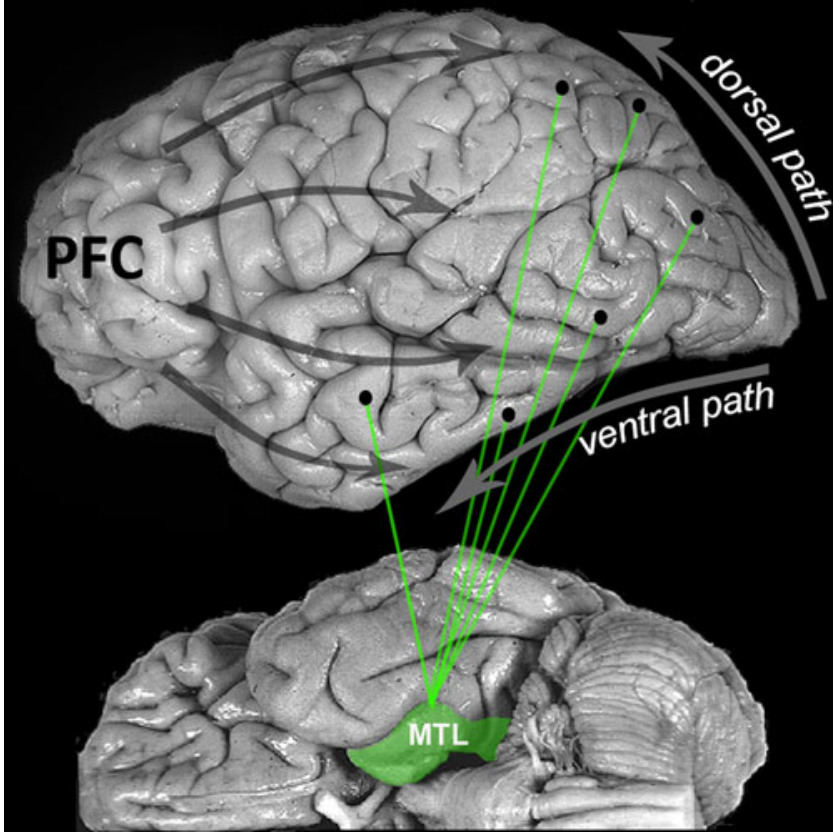

Fig. 1 Critical components of episodic encoding include (1) bottom-up sensory features, such as those established by visual processing (e.g., dorsal and ventral paths), (2) top-down control implemented by projections from the prefrontal cortex (PFC) to posterior cortical sites that represent event features, and (3) medial temporal lobe (MTL) bindings that link and store coactive event features as an episodic memory ensemble

rapid associative process akin to long-term potentiation (Lynch, Rex, \& Gall, 2007; Morris, 2006). MTL bindings link together episodic features and facilitate reactivation or replay of these ensembles after encoding. According to consolidation theories, episodic memories are ultimately represented as neocortical ensembles of episodic features (Shimamura \& Wickens, 2009; Squire et al., 1989).

\section{Role of the prefrontal cortex in episodic retrieval}

Episodic retrieval typically occurs without the benefit of rich sensory cues. In everyday situations, a cue may simply be, "What did you do last weekend?" For such sparse cues, successful retrieval depends on an active search of relevant event features, such as people and places encountered during an event. From accessing a few features, a fuller reinstatement of event features - that is, a recollection of an episodic event-can be achieved. In computational terms, this process is defined as pattern completion (Norman \& O'Reilly, 2003). Prior to the advent of functional neuroimaging (e.g., PET, fMRI), the neural correlates of episodic retrieval were not well defined. Retrieval deficits are common in patients with widespread neocortical damage, such as in Alzheimer's disease or following traumatic brain injury, but these disorders also include impairment in other cognitive domains, such as deficits in attention, new learning, language, and reasoning (Bruen, McGeown, Shanks, \& Venneri, 2008; Kopelman, 1989). Patients with PFC lesions exhibit mild to moderate impairments in episodic retrieval (Hwang et al., 2007; Mangels, Gershberg, Shimamura, \& Knight, 1996), but PFC lesions also cause broad deficits in executive control or metacognitive processes (Janowsky, Shimamura, \& Squire, 1989; Shimamura, 2008). Analyses of event-related potentials (ERPs) suggested a frontal-parietal circuit involved in episodic retrieval (Rugg \& Wilding, 2000); however, limitations in the spatial resolution of ERPs have made it difficult to identify specifically the neocortical generators of such activity.

Early PET and fMRI studies used blocked designs in which sets of recognition test items consisting primarily of old items were compared with sets that consisted primarily of new items (Kapur et al., 1995; Rugg, Fletcher, Frith, Frackowiak, \& Dolan, 1996; Tulving et al., 1994). These analyses revealed significantly greater activation in the dorsolateral PFC when "old" blocks were compared with "new" blocks. At that time, it was unclear whether retrieval-related activation was based on successful episodic recollection or simply associated with setting a retrieval "mode" throughout a block of old items. Despite this limitation, early neuroimaging findings suggested a critical role of the PFC in episodic retrieval.

With the advent of event-related fMRI, PFC activity has been confirmed as a robust neural correlate of successful episodic retrieval (Buckner et al., 1998; Dobbins, Foley, Schacter \& Wagner 2002; Simons \& Spiers 2003). Numerous findings have shown that the PFC facilitates top-down selection, control, and updating of relevant episodic features (see Cabeza \& St. Jacques, 2007; Ranganath, Johnson, \& D’Esposito, 2000; Rugg, Fletcher, Chua, \& Dolan, 1999). Analyses of regions within the PFC suggest that the ventrolateral PFC is involved in the maintenance of item features, semantic access, and initiating retrieval, whereas the dorsolateral PFC is involved in updating and manipulating retrieved features and in more complex or higher-order goaldirected retrievals (see Badre, 2008; Bunge, 2004; Christoff \& Gabrieli, 2000; Raposo, Han, \& Dobbins, 2009; Wagner, 2002). The MTL also contributes to retrieval, presumably by accessing and activating event features through relational bindings (Eldridge, Engel, Zeineh, Bookheimer, \& Knowlton, 2005; Gilboa et al., 2005; for theoretical analyses, see Moscovitch, Nadel, Winocur, Gilboa, \& Rosenbaum, 2006; Shimamura, 2010).

\section{Parietal contributions to episodic retrieval}

In addition to PFC and MTL involvement, it is now appreciated that the posterior parietal cortex (PPC) plays a 
significant role in episodic retrieval. On recognition tests, PPC regions are active when individuals correctly identify items as old (hits), as compared with correctly identifying items as new (correct rejections, or CRs). This increased activation of hits $>$ CRs is termed the successful retrieval effect. Although early neuroimaging studies occasionally reported retrieval-related PPC activity, its nature and importance has only recently been appreciated. The PPC is now considered one of the most, if not the most, active region during the successful retrieval of episodic memory (for reviews, see Cabeza, Ciaramelli, Olson, \& Moscovitch, 2008; Shannon \& Buckner, 2004; Vilberg \& Rugg 2008; Wagner, Shannon, Kahn, \& Buckner, 2005).

The successful retrieval effect has been observed using a variety of stimuli types, such as visual and spoken words, pictures, faces, natural sounds, and music clips (Cansino, Maquet, Dolan, \& Rugg, 2002; Dobbins \& Wagner, 2005; Guerin \& Miller, 2009; Henson, Rugg, Shallice, Josephs, \& Dolan, 1999; Klostermann, Kane \& Shimamura 2008; Klostermann, Loui, \& Shimamura, 2009; Leube, Erb, Grodd, Bartels, \& Kircher, 2003; Shannon \& Buckner, 2004; Svoboda, McKinnon, \& Levine, 2006). It has also been observed under various test conditions and manipulations, such as recognition memory, cued recall, source memory, confidence judgments, and motor responses (see de Zubicaray et al., 2007; Shannon \& Buckner, 2004; Vilberg \& Rugg 2008; Wagner et al., 2005). For example, Shannon and Buckner demonstrated that PPC activity does not depend on specific motor intentions. They found retrieval-related PPC activity when subjects responded only to old items or only to new items. Thus, regardless of the response intention, PPC activity was correlated with successful retrieval (hits $>$ CRs). Incorrect recognition trials (false alarms, misses) are not generally analyzed, due to the limited number of such responses. However, when these responses have been assessed, PPC activity is greater for false alarms (responding "old" to new items), as compared with CRs, suggesting that PPC activity is an index of subjective feelings of remembering, even when such feelings are invalid (Kahn, Davachi, \& Wagner, 2004; Slotnick \& Schacter, 2004; Wheeler \& Buckner, 2003).

Three broad PPC regions have been associated with the successful retrieval effect: (1) the dorsal PPC (dPPC), which includes the superior parietal lobule (SPL) and intraparietal sulcus (IPS); (2) the ventral PPC (vPPC), which includes the supramarginal gyrus (SMG), angular gyrus (AnG), and temporal-parietal junction (TPJ); and (3) the medial PPC (mPPC), which includes the precuneus, retrosplenial cortex, and posterior cingulate cortex. Although retrieval-related activations in these regions have been consistently observed, it is the vPPC that appears most directly associated with episodic retrieval and source recollection (see Cabeza et al., 2008; Vilberg \& Rugg, 2008). For example, studies have shown differential activations for recollection versus familiarity judgments. Recollection depends on the retrieval of specific source features, whereas familiarity refers to a nonspecific feeling of knowing. Recollection-based activations have been observed using the remember/know $(\mathrm{R} / \mathrm{K})$ paradigm, in which items judged as "old" are further assessed as having a strong recollection of source features ( $\mathrm{R}$ items) or only a general noninformative feeling of familiarity ( $\mathrm{K}$ items). Recollection-based activations are also assessed in source memory tests, since items with correctly identified source features are compared with items judged as old but with incorrect source memory. It is the VPPC that monitors the degree of recollection-based retrieval, since this region is particularly active for $\mathrm{R}>\mathrm{K}$ items and correct source $>$ incorrect source items (Cansino et al., 2002; Daselaar, Fleck, \& Cabeza, 2006; Dobbins et al., 2002; Kahn et al., 2004; Wheeler \& Buckner, 2004; Yonelinas, Otten, Shaw, \& Rugg, 2005). In addition, vPPC responds more strongly to high-confident old and new judgments, as compared with low-confident judgments (see Cabeza et al., 2008).

Recent meta-analyses have confirmed the dissociation between dPPC and vPPC activation (Cabeza et al., 2008; Hutchinson, Uncapher \& Wagner 2009; Spaniol et al., 2009; Vilberg \& Rugg, 2008; Wagner et al., 2005). Whereas the APPC is associated with familiarity-based activity or with nonspecific attentional processes, it is the vPPC that is associated with episodic recollection. Activity in the APPC is greater for low-confident responses than for high-confident responses, suggesting that this area is recruited when effortful or more difficult judgments are required. In such cases, it may be that more extensive topdown control must be initiated as a result of poorly reinstated source features during retrieval. With respect to the medial PPC, less is known about the specific conditions under which recollection-related activity occurs. There is evidence that both recollection-based and familiarity-based activity occurs within the medial PPC, although the segregation of these activations is less apparent (see Vilberg \& Rugg, 2008).

Meta-analyses of encoding activity suggest that the vPPC and dPPC respond quite differently during the initial encoding of episodic features (Spaniol et al., 2009; Uncapher \& Wagner, 2009). In subsequent memory analyses, activations during encoding are sorted in terms of those items that are recognized and those that are not recognized in a later memory test (see Paller \& Wagner, 2002). Uncapher and Wagner found that $85 \%$ of the positive subsequent memory effects in the lateral PPC occurred in dorsal regions (in the IPS or dorsal to it). Thus, increased activity in the dPPC during encoding, such as regions along the dorsal visual path, is a strong predictor of successful retrieval at a later time. The vPPC is generally less responsive as a marker for encoding success than it is 
as a marker for retrieval success (Spaniol et al., 2009). In fact, negative subsequent memory effects have been observed in the vPPC. In these cases (see Uncapher \& Wagner, 2009), vPPC activity during encoding, if anything, had a deleterious influence on subsequent recognition performance. These findings need to be addressed within the context of the important role that the vPPC plays during retrieval.

For both the vPPC and dPPC, retrieval-related activity is typically left lateralized, although bilateral activity has also been observed (Guerin and Miller 2011; Henson et al., 1999; Shannon \& Buckner, 2004; Vilberg \& Rugg, 2008). Indeed, left-lateralized PPC activity has been observed for both nonverbal information (faces) and nonvisual information (see Guerin \& Miller 2011; Klostermann et al., 2008; Shannon \& Buckner, 2004). For example, Klostermann et al. (2008) found left-lateralized PPC activation for auditory presentations (spoken words). In that study, subjects kept their eyes closed so that bottom-up visual processing could not drive memory-related responses. If, however, PPC activity monitors the retrieval of event features, it seems reasonable that right-lateralized PPC activity would be observed for items or features encoded by the right hemisphere. To date, there is only one study in which right-lateralized successful retrieval effects have been observed (Klostermann et al., 2009). In that study, nonWestern, nonverbal music clips were used as stimuli. These stimuli are known to be encoded and represented primarily by higher-order auditory regions in the right hemisphere. For these stimuli, successful retrieval effects were observed only in the right PPC. No left-hemisphere PPC activity was found, even when statistical threshold levels were lowered. Such findings suggest that retrieval-related PPC activity is associated with the cortical regions involved in encoding and storing episodic features. The preponderance of leftlateralized effects is likely due to the use of verbal or semantic retrieval processes that are known to be represented in the left hemisphere.

\section{To what extent do patients with parietal lesions exhibit episodic retrieval deficits?}

Patients with PPC lesions exhibit a variety of cognitive disorders, including deficits in visuospatial attention, sensorimotor integration, and verbal short-term memory (see Baldo \& Dronkers, 2006; Egly, Driver, \& Rafal, 1994; Olson \& Berryhill, 2009; Pause, Kunesch, Binkofski, \& Freund, 1989; Warrington \& Shallice, 1969). Such patients do not exhibit severe impairment in learning and memory, certainly not to the same degree as patients with MTL or PFC lesions. Yet recent studies, motivated by neuroimaging findings linking PPC activity to episodic retrieval, have shown some impairment in episodic recollection. Berryhill, Phuong, Picasso, Cabeza, and Olson (2007) assessed episodic memory in two patients with bilateral PPC lesions. Both patients exhibited visual simultagnosia, an impairment in the ability to attend to multiple visual elements or objects. Neither patient exhibited the kind of deficits in new learning ability observed in patients with organic amnesia, although both exhibited impoverished recollections when asked to recall detailed autobiographical events from periods in their lives. Interestingly, the patients exhibited good cued recall when given specific probes to episodic features. The authors suggested that memory impairment was analogous to their impairments in visual attention. That is, these patients appeared to exhibit a kind of memory simultagnosia in which multiple episodic features could not be attended or reinstated together at the time of retrieval.

Source memory impairment has been observed in patients with MTL lesions (Shimamura and Squire 1991) and in patients with PFC lesions (Janowsky et al., 1989). Simons et al. (2008) assessed source memory in six patients with unilateral PPC lesions (three left- and three right-sided lesions). At study, stimuli (words and famous faces) were initially presented with one of two questions: "Does the stimulus relate more to entertainment or politics?" or "Does the stimulus seem pleasant or unpleasant?" At test, subjects were shown a study item and were asked to determine whether the item was previously presented with the pleasantness/ unpleasantness question or with the entertainment/politics question. As a group, PPC patients did not exhibit significant source recognition impairment, although one left-lesioned patient performed more than two standard deviation units below the control mean on the source test for famous faces. The authors suggested that this patient might have damage that extended into the frontal lobes. In a follow-up study (Simons, Peers, Mazuz, Berryhill, \& Olson, 2010), source memory was assessed in six patients with unilateral lesions and in the two patients with bilateral PPC lesions studied by Berryhill et al. (2007). Both unilateral and bilateral PPC patients performed as well as control subjects on tests of source memory, although the bilateral patients were reliably less confident about their responses.

In another study, Davidson et al. (2008) assessed both source recognition and remember/know judgments in patients with PPC lesions (four left- and one right-sided lesions). Patients were presented novel definitions (e.g., a talkative featherbrain - parakeet), with half of these definitions presented visually and the other half presented auditorily. At test, subjects were asked to provide answers to both old and new definitions (cued recall test). They were then asked to determine whether the definition was an old or new one (recognition test). For old definitions (e.g., a talkative featherbrain?), if they provided the correct answer, they were asked if they originally had seen or heard the 
definition before (source test). Finally, participants were asked whether they had a strong recollection (remember) or less strong memory (know) of the prior presentation of the definition (remember/know test). PPC patients were significantly impaired on the old/new recognition test and elicited fewer remember responses than did controls. They were not significantly impaired on the cued recall or source tests (although one patient scored nearly two standard deviation units below the control group on the source test; .62 vs .38, $S D=.14)$.

In a separate analysis, Davidson et al. (2008) assessed a PPC patient on the Deese-Roediger-McDermott (DRM) false recognition paradigm. In this task, semantic associates (e.g., snore, night, dream) are presented for study, and old/ new recognition performance is assessed for old items and for false recognition of semantically related lures (e.g., sleep). This patient elicited fewer false recognitions to the lures and fewer hits than did control subjects, suggesting a reduced confidence in recognition judgments. To corroborate these findings, Drowos, Berryhill, Andre, and Olson (2010) administered the DRM paradigm to the two bilateral PPC patients assessed by Berryhill et al. (2007) and Simons et al. (2010). These patients elicited considerably fewer false recognitions of semantically related lures than did controls. Moreover, they were less confident than controls for true hits. Such findings are not unlike those observed in patients with MTL lesions (see Schacter, Verfaellie,\& Koutstaal, 2002), whereas patients with PFC lesions have been shown to exhibit higher rates of false recognitions to semantically related lures (Budson et al., 2002; Schacter, Curran, Galluccio, Milberg \& Bates 1966).

Finally, Haramati, Soroker, Dudai and Levy (2008) assessed a group of 17 patients with unilateral lesions resulting from cerebrovascular accidents (6 left- and 11 right-hemisphere lesions). All patients had PPC lesions, although all had lesions in other cortical regions. Lesionbehavior correlations and voxel-based lesion symptom mapping (VLSM) analyses were applied as ways to relate old/new recognition memory performance (for pictures, words, and sounds) to lesion site. Recognition memory impairment was correlated with right-hemisphere lesions in the PFC and temporal cortex. Patients with left-hemisphere lesions did not exhibit significant recognition memory impairment, but correlational analyses did reveal significant associations between performance and lesions in the superior occipital cortex (pictures) and AnG (words). VLSM analyses showed that regions within the PFC, temporal cortex, and vPPC (AnG, SMG) were associated with recognition memory performance. Although PPC regions were implicated in these analyses, the authors suggested that other regions (PFC, temporal cortex) were more involved in mediating recognition memory performance than were parietal regions. A similar conclusion was made by Rossi et al. (2006), who used repetitive transcranial magnetic stimulation (rTMS) to produce transient disruption of PPC regions during memory retrieval. They found that rTMS did not significantly disrupt memory performance, although it did slow attentional processes.

Taken together, patients with PPC lesions exhibit some deficits in episodic memory, although certainly not to the same degree as patients with MTL or PFC lesions. In particular, they appear less able to formulate rich, contextually based recollections of prior experiences. The reduced experience of strong episodic retrieval is best reflected by their reduced remember judgments on tests of recognition memory. Also, reduced false recognition performance on the DRM paradigm suggests an impairment in relating items within a contextually bound experience. Normal or near-normal performance on tests of item and source recognition suggests that the ability to recognize specific features or associate them with past experiences is intact.

\section{The role of the posterior parietal cortex in other cognitive domains}

Regions within the PPC have been associated with other cognitive processes, particularly those related to visuospatial processing (for reviews, see Corbetta \& Shulman, 2002; Silver \& Kastner, 2009). A critical PPC region for spatial attention is the IPS. In many studies, the IPS is active in tasks involving visual selective attention (Corbetta, Kincade, Ollinger, McAvoy \& Shulman 2000; Serences \& Yantis, 2007; Yantis et al., 2002). It is thought that the IPS acts in conjunction with PFC regions and bottom-up sensory paths to initiate control of visuospatial processing. More complicated visuospatial tasks have been used to define functional dissociations within regions of the lateral PPC. Whereas visual switching activates the posterior IPS (extending into occipital regions), response switching activates other PPC areas, including the anterior IPS, superior parietal lobule, and SMG (Rushworth, Paus \& Sipila 2001; see also Rounis, Yarrow \& Rothwell 2007; Serences \& Yantis, 2006). These findings suggest that the PPC is involved in visuospatial attention and in integrating stimuli with responses.

Other findings suggest that the lateral PPC is involved in working memory processes, such as engaging, maintaining, and rerouting visuospatial, phonological, or motor processes. For example, the IPS is active when subjects are asked to maintain or manipulate in working memory visually presented words or faces (Majerus et al., 2007). The left vPPCspecifically, the SMG - has been associated with phonological working memory. For example, Paulesu, Frith and Frackowiak (1993) assessed trials on which subjects were asked to rehearse verbally a set of visually presented letters. This task was compared with others that did not involve verbal 
rehearsal, such as a visual imagery or a rhyming judgment task. Verbal rehearsal activated both the SMG and the inferior frontal gyrus (Broca's area), suggesting that these two regions form a circuit for the control and maintenance of phonological information (see also Celsis et al., 1999).

The PPC has also been central in integrating multimodal spatial information (Renier et al., 2009) and integrating sensory input with motor programs (Andersen \& Buneo, 2002). These multisensory processes exemplify the integrative aspect of this brain region and have led to this area being referred to as representing "intentional maps" or "mirror neurons" (Andersen \& Buneo, 2002; Cattaneo \& Rizzolatti, 2009). For example, Buccino et al. (2001) observed lateral vPPC activity while subjects watched videotapes of individuals performing actions (e.g., biting an apple, kicking a ball). In a broader sense, findings of intentional maps are related to another cognitive domainthe notion of theory of mind, which refers to the ability to consider another's perspective or intended action. Saxe and colleagues have shown that the TPJ in the vPPC is active on tasks involving theory of mind (Saxe \& Kanwisher, 2003; Young \& Saxe, 2008; see also Aichhorn et al. 2009; Buckner \& Carroll, 2007). In these studies, performance depends on the consideration of another's actions, thoughts, or feelings.

In cross-task fMRI analyses, two tasks are presented during the same scanning session to determine the degree of overlap in activations between them. Sestieri, Shulman and Corbetta (2010) used a visual search task and an item recognition task and found that activity in the PPC was largely segregated between the two tasks. Recognitionrelated activations were observed in the vPPC (AnG) and $\mathrm{mPPC}$ (precuneus, posterior cingulate cortex), whereas search-related activations were observed in the dPPC (IPS). Two studies have used cross-task analyses to assess regional overlap between episodic retrieval and maintaining information in working memory (Cabeza, Dolcos, Graham, \& Nyberg, 2002; Ranganath et al., 2003). In these studies, regions within the vPPC, mPPC, and PFC were more active during episodic retrieval than during working memory trials. Moreover, there was more regional overlap in activity between episodic retrieval and working memory processes.

Several studies have used cross-task analyses to assess autobiographical retrieval and perspective taking (e.g., theory of mind, future planning). Spreng and Grady (2009) found correlative activity within the default mode network (PFC, vPPC, mPPC) for autobiographical retrieval, thinking about the future, and theory of mind. In another study (Rabin et al., 2010), subjects were asked to reexperience a past event cued by a family photo (autobiographical recollection) or generate a novel event cued by a stranger's photo (i.e., perspective taking).
Overlapping activity occurred in the PFC (ventrolateral, polar regions), $\mathrm{mPPC}$ (posterior cingulate, retrosplenial cortex), MTL (hippocampus, perirhinal cortex), and lateral temporal cortex (middle temporal gyrus, temporal pole). Greater activity for recollection versus perspective taking occurred in the perirhinal cortex and medial PFC, whereas relatively greater activity for perspective taking was observed in the vPPC (AnG, SMG, TPJ) and ventrolateral PFC. These findings point to considerable overlap in activity occurring between autobiographical retrieval and perspective taking, suggesting that $\mathrm{VPPC}$ activity may be more broadly involved in integrating contextual features than being specifically related to autobiographical retrieval.

\section{Theoretical approaches to the role of the PPC in episodic retrieval}

It is clear from this review that PPC activity is associated with episodic retrieval, as well as with other cognitive processes. How can one explain such broad-based PPC activity? One possibility is that neural activity is actually domain specific and each domain has its own segregated region within the PPC. On the other hand, seemingly diverse processes may share common mechanisms and, hence, common neural circuits. To summarize, any theory of retrieval-related vPPC activity must account for the following findings:

1. In studies of item recognition memory, $\mathrm{vPPC}$ activity is greater for hits than for correct rejections (hits $>\mathrm{CRs}$ ).

2. vPPC activity is greater for false alarms than for correct rejections (FA $>$ CRs).

3. vPPC activity is greater for high-confident hits than for low-confident hits (high hits $>$ low hits).

4. vPPC activity is greater for high-confident correct rejections than for low-confident correct rejections (high CRs $>$ low CRs).

5. In studies of the remember/know paradigm, vPPC activity is greater for remember (i.e., recollection) responses than for know (i.e., familiarity) responses $(\mathrm{R}>\mathrm{K})$.

6. In studies of source recognition memory, vPPC activity is greater for items with correct source memory than for items with incorrect source memory (correct source $>$ incorrect source).

7. Retrieval-related vPPC activity is usually leftlateralized or bilateral, although specifically right vPPC activity was observed during successful retrieval of music stimuli.

8. At encoding, vPPC activity is minimally correlated and sometimes negatively correlated with subsequent retrieval. 
9. Patients with PPC lesions do not exhibit significantly impaired source recognition or probed cued recall, but they elicit fewer recollective (i.e., remember) responses, fewer recognition hits, fewer false recognitions to semantically related lures, and reduced confidence ratings for hits. They also elicit less detail or richness in recalling autobiographical memories.

10. vPPC activity is associated with task demands in cognitive domains other than episodic retrieval, including spatial attention, multisensory integration, visual and phonological working memory, sensorimotor integration, theory of mind, and future planning.

Three theories have been proposed to account for retrieval-related activity in the PPC. The mnemonic accumulator theory (M-ACC; Wagner et al., 2005; see also Huijbers, Pennartz, \& Daselaar, 2010) suggests that the PPC acts as a retrieval monitor that increments or accumulates the amount of information retrieved in making a recognition decision. This theory is based on Ratcliff's diffusion model of recognition memory (Ratcliff, 1978; Ratcliff \& Starns, 2009). M-ACC accounts for both recollection-based effects and subjective experiences, because the accumulation of evidence for a recognition decision could be valid (leading to hits) or spurious (leading to false alarms). Indeed, M-ACC is directed toward the monitoring of any retrieved information and suggests that the PPC is not necessarily tied to specific features of an episodic event.

The episodic buffer theory (E-BUFF; Vilberg \& Rugg, 2008) suggests that PPC activity is tied rather closely to episodic features. This theory draws on Baddeley's (2000) revised working memory model, in which an episodic buffer acts as a temporary store of multimodal event features. According to E-BUFF, this short-term memory process is separate from long-term memory representations and is particularly tied to recollective (as opposed to familiarity) processes. It is proposed that the $\mathrm{vPPC}$ acts as an episodic buffer by holding online source features during retrieval (Vilberg \& Rugg, 2008). Whereas the vPPC acts as an episodic buffer, the dPPC is presumed to reflect nonspecific processing or processes involved in both recollection and familiarity.

Both M-ACC and E-BUFF theories offer a cognitive component-accumulator or episodic buffer-that relates PPC activity to an aspect of episodic retrieval. Although both theories define a particular role for the PPC during retrieval, neither suggests how PPC activity is incorporated into a larger network involved in episodic retrieval. Specifically, neither purports to explain how the PPC interacts with PFC and MTL components. The attention to memory theory (AtoM; Cabeza et al., 2008; Ciaramelli, Grady, \& Moscovitch, 2008) does embed PPC activity within a broad neural network that includes PFC and MTL. AtoM is based on a theory of selective attention proposed by Corbetta and Shulman (2002). According to AtoM (Cabeza et al., 2008; Ciaramelli et al., 2008), the dPPC is part of a frontoparietal circuit involved in top-down or goaldirected retrieval processes. These top-down processes help define search strategies and reroute memory access toward successful retrieval. The vPPC acts as a bottom-up feed of activated memories. These bottom-up activations are initiated by sensory cues or by episodic features retrieved by way of MTL bindings. According to AtoM, the dPPC is tied more closely to the PFC in driving top-down attention to salient retrieval paths, whereas the $\mathrm{VPPC}$ is tied more closely to the MTL in activating episodic features.

AtoM defines a neural circuitry that is linked directly to attention circuits derived by the Corbetta and Shulman (2002) model. To assess AtoM, a meta-analysis of regions associated with retrieval and attention was performed (Hutchinson et al., 2009). Findings suggested greater segregation of retrieval and attention activations than convergence. In particular, bottom-up attention-related activations in the vPPC did not overlap with recollectionrelated activations. Across studies, attention-related activity occurred in more anterior regions of the vPPC (SMG, TPJ), whereas recollection-related activity was centered in the AnG. Thus, although AtoM offers a neural circuit that integrates attention and retrieval mechanisms, lack of regional overlap across these two mechanisms appears to suggest more differences than similarities between episodic retrieval and visual attention (see also Sestieri et al., 2010).

\section{Cortical binding of relational activity (CoBRA): A new theory}

Episodic memory involves broad cortical networks necessary for encoding, representing, and ultimately retrieving past events and experiences. Proposed here is a theory of episodic memory in which the vPPC contributes to the cortical binding of relational activity (CoBRA). On this view, the vPPC acts as a convergence zone that binds episodic feature ensembles within the neocortex. This associative or integrative process begins soon after encoding and continues long after the initial event. The vPPC is well situated to bind episodic features, since it is classically viewed as the "parieto-temporo-occipital crossroads" (Critchley, 1966, p. 197). Anatomical studies of putatively homologous regions in nonhuman primates (area 7a or PG) have shown that the $\mathrm{VPPC}$ is intricately connected to regions in the dorsal visual path (dPPC, extrastriate areas), ventral visual path (superior temporal sulcus, inferotemporal cortex), MTL (parahippocampal gyrus), and PFC (dorsolateral PFC) (Andersen, Asanuma, Essick, \& Siegel, 1990; Cavada \& 
Goldman-Rakic, 1989; Pandya \& Seltzer, 1982). As such, the VPPC is geographically central and well connected to many neocortical regions, thus giving it the advantage of establishing intermodal links across diverse event features, such as object-based features, spatial features, acoustic features, and verbal/semantic features. A critical prediction of CoBRA is that tasks that demand the reinstatement of intermodal features from disparate neocortical sites, such as voices with faces or objects with spatial locations, will depend more on vPPC bindings than will tasks that depend on within-modality or within-object associations.

Cortical binding within the vPPC works in conjunction with other known processes. In particular, there is a functional relationship between $\mathrm{VPPC}$ and MTL bindings. According to consolidation theories, such as hierarchical relational binding theory (hRBT; Shimamura, 2010; Shimamura \& Wickens, 2009; see also Cohen \& Eichenbaum, 1993; Squire \& Zola, 1998), the MTL binds event features at the time of encoding and later participates in the reactivation, or "replay," of these features during reminiscence. It is thought that MTL-driven reactivations help to create neocortical links between episodic features, so that ultimately MTL bindings become less or not at all involved during retrieval of remote (i.e., fully consolidated) memories. Extant consolidation theories do not specify exactly what happens within the neocortex to establish episodic memories as encapsulated neocortical representations. According to CoBRA, the reactivation of episodic memories, which is driven by MTL bindings, leads to the creation of neocortical bindings within the vPPC, thus establishing relational links between geographically disparate episodic features. Thus, CoBRA refines consolidation theory by outlining its final stage, which is to bind episodic memories completely within the neocortex. The proposal here is that such inter-feature links are reinforced by bindings within the vPPC.

One may ask, why propose a process within the neocortex that is functionally similar to one attributed to the MTL? It is true that in most cases, episodic recollectionthat is, the retrieval of an encapsulated ensemble of episodic features (as in reminiscing about a past event) -will likely depend on both MTL and VPPC bindings. MTL bindings, as a result of their being fully established at the time of initial encoding, are more critical than vPPC bindings for the retrieval of recent memories. Yet it is well established that remote autobiographical memories can be retrieved by patients with MTL lesions (Bayley, Hopkins, \& Squire, 2003; Squire, Haist, \& Shimamura, 1989), although there is still controversy over whether this ability is fully intact (see Moscovitch et al., 2006). In any event, the MTL is not necessary for the retrieval of remote memories, and it is argued here that the vPPC takes on the role by establishing links within the neocortex. As was stated earlier, the vPPC is well situated as a convergence zone in that it is geographically central to regions known to represent event features (e.g., dorsal path, ventral path, superior temporal lobe). On this view, the MTL and vPPC function together to bind episodic features, although they have complementary roles. MTL bindings occur immediately during encoding and become less involved as time passes, whereas VPPC bindings are created after encoding, depend on initial MTL bindings, and become more involved as time passes. The close tie between MTL and $\mathrm{vPPC}$ processes is confirmed by anatomical and functional connectivity analyses. Clower, West, Lynch, and Strick (2001) showed that the vPPC (area 7a) receives a strong disynaptic input from the CA1 region of the hippocampus via the parahippocampal gyrus. In a connectivity analysis of fMRI data, Vincent et al. (2006) identified significant coherent resting state activity between the MTL and $\mathrm{VPPC}$.

Episodic retrieval is typically initiated by sparse cuing (e.g., What did you do last weekend? Was this word presented to you an hour ago?). As is shown in Fig. 2, during retrieval, the PFC initiates top-down search of episodic features. According to hRBT (Shimamura, 2002, 2010), MTL bindings available at the time of retrieval assist in the reinstatement of event features by way of pattern completion mechanisms (Norman \& O'Reilly, 2003). According to CoBRA, the vPPC also contributes to the successful retrieval of episodic memory,since relational

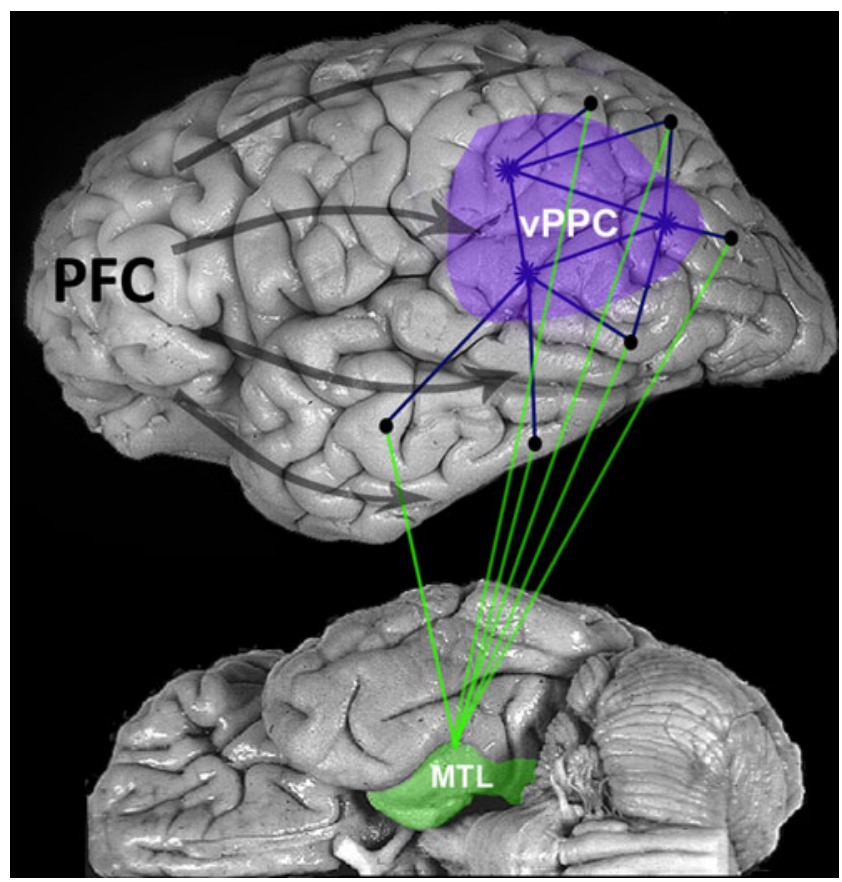

Fig. 2 According to CoBRA, episodic retrieval is initiated by topdown prefrontal cortex (PFC) activation of event features, which are facilitated by MTL relational bindings. The vPPC acts as a convergence zone that links event features within the neocortex. These links form after encoding through reactivation or replay of episodic ensembles and facilitate reinstatement of these ensembles during retrieval 
bindings within this region also facilitate the reactivation of multimodal features linked to a past event. On this view, the vPPC acts as a way of offloading the reliance of MTL bindings. As episodic memories become reactivated over time, such as through reminiscence, vPPC bindings become more involved in their retrieval. Episodic recollection, however, depends not just on vPPC bindings, but also on a broad neural circuit that includes the PFC, MTL, and cortical sites that store event features. Thus, it is acknowledged that none of these brain regions is individually sufficient or necessary for the retrieval of episodic memories, yet each plays a particular role in the "re-collection" of past events and experiences.

\section{Implications of CoBRA theory}

CoBRA defines the role of the vPPC as a process that integrates features of episodic memories as encapsulated, cortically bound ensembles. Some aspects of episodic retrieval may depend minimally on vPPC bindings because they draw on memory for individual event features or general feelings of familiarity. For example, answering the question, Did you have spaghetti for dinner yesterday? may depend only on a vague warmth value or sense of familiarity. However, answering the question, What did you eat for dinner yesterday? depends on the reinstatement of an encapsulated ensemble of event features that represent a specific time, place, and action. Such recollection-based responses depend on successful binding and retrieval of multiple episodic features, and it is this kind of response that depends most on vPPC bindings. As vPPC bindings integrate event features into multimodal ensembles, it is predicted that this region is particularly involved during the successful retrieval of contextually bound episodic memories. Thus, findings of the role of the vPPC in (1) highly confident hits (high-confident hits $>$ low-confident hits), (2) recollectionbased retrievals ( $\mathrm{R}>\mathrm{K}$ responses), and (3) successful source memory (correct $>$ incorrect source memory) are consistent with CoBRA.

It is acknowledged that this binding process is not always exact, and thus spurious bindings could be formed and attach themselves to episodic ensembles during reactivation or replay of past events. Such spurious bindings would form the basis for the experience of false recognitions and déjà vu. Thus, a subjective experience of recollection, whether valid or not, would garner vPPC activity. CoBRA predicts that false recognitions would generate $\mathrm{vPPC}$ activity as in the case of FA $>$ CRs. To explain the finding of increased vPPC activity for highconfident $\mathrm{CRs}>$ low-confident CRs, it must be presumed that to judge confidently that an item was not a part of a past event (i.e., a truly novel item), one must have first successfully retrieved an encapsulated episodic memory and then determined that an item was not part of the memory. This proposition is akin to the phenomenon of recall to reject (Clark \& Gronlund, 1996). Yet given this interpretation, it must be argued that high-confident hits (knowing that an item was definitely presented during the study phase) depend more on vPPC binding than do highconfident CRs (knowing that an item was definitely not presented). Further analyses are needed to confirm this assumption. For example, to remember that a certain person was definitely at one's birthday party is predicted to require more episodic feature binding than remembering that a certain person was definitely not at the same celebration. Indeed, the presence of the person could elicit multiple event features (i.e., source memory) and enhance episodic recollection.

According to CoBRA, vPPC bindings link episodic features that are specific to a past event or experience. As such, these bindings must be closely tied to the cortical sites where such features are stored. Thus, vPPC bindings must be ones that link event features in adjacent or closely connected regions. As was stated previously, this region is well situated to link event features stored in the dorsal visual path, ventral visual path, and lateral temporal lobe. A corollary of this principle is that episodic features that are encoded primarily by one cerebral hemisphere should be linked by vPPC bindings in that same hemisphere. This prediction is supported by the findings of Klostermann et al. (2009), in which retrieval-related activity in the right vPPC was found for stimuli (unfamiliar music clips) known to be processed in the right hemisphere. On this view, the frequently observed finding of left-lateralized vPPC activity (for words, pictures, and faces) is attributed to vPPC bindings of verbal or semantically based features known to be represented primarily in the left hemisphere.

In many respects, vPPC bindings are an integral part of episodic representations. That is, such bindings link episodic features to each other. Whenever one experiences a recollection of a past event, whether generated by effortful top-down search or simply as an episodic memory that pops into mind, these vPPC bindings should be activated. On this view, vPPC activity should be observed during implicit retrieval, as well as explicit retrieval, although perhaps less so, since the latter should involve more extensive use of feature bindings. This prediction is supported by a recent study in which vPPC activity was observed for recently studied words later encountered during an implicit lexical decision task (Elman \& Shimamura, in press).

During encoding, the establishment of episodic memories depends on (1) bottom-up activation of sensory features, such as those along the dorsal and ventral paths, (2) top-down PFC processes involved in selecting and maintaining relevant event features, and (3) MTL binding 
of features as an episodic ensemble (see Fig. 1). According to CoBRA, vPPC bindings develop after the encoding event by reactivations of event features. Thus, it is not expected that $\mathrm{VPPC}$ activity would be particularly strong during episodic encoding, a finding consistent with metaanalyses of encoding-related activity (Spaniol et al., 2009; Uncapher \& Wagner, 2009). It is not known how long after encoding vPPC bindings begin to develop. It may be that such bindings begin to develop during the moments just after stimulus encoding, such as during working memory rehearsal or replay of recent events. Findings of vPPC activity during the maintenance of information in working memory (Cabeza et al., 2002; Ranganath et al., 2003) suggest that $\mathrm{vPPC}$ bindings may begin to form immediately after encoding.

Evidence from studies of the default network (Buckner, Andrews-Hanna, \& Schacter, 2008; Spreng \& Grady, 2009) suggest that the vPPC is part of a neural circuit involved in internally generated thought processes (e.g., thinking, mind wandering, planning, remembering). During such offline moments, perhaps even immediately after a stimulus is presented, internally generated rehearsals begin to establish vPPC bindings. During such reactivations, the MTL and vPPC may work together to "consolidate" bindings within the neocortex. The finding of negative subsequent memory effects in the vPPC (and in other regions associated with the default network) may be the result of the brain's working to encode event features perceptually, rather than binding them via vPPC links. In cross-task analyses, Sestieri et al. (2010) found evidence for a "reciprocal dynamic competition" for attentional resources between perception and memory.

To the extent that CoBRA defines the final stage of consolidation by creating durable and long- lasting neocortical representations, it is predicted that the contribution of the vPPC in episodic retrieval will increase with the age of the memory. Although retrieval-related activity in the vPPC can be observed following rather short delays, it may be that with time (and more replay), long-lasting episodic memories become fully bound by vPPC bindings. CoBRA predicts that repetitive retrieval or replay of an episodic memory should incrementally increase vPPC binding. Interestingly, Saletin, Goldstein \& Walker (in press) found that sleep-spindle activity recorded over the left parietal cortex during a nap between study and test phases was correlated with subsequent retrieval. Such findings suggest that a function of sleep-enhanced consolidation may be to facilitate vPPC binding after encoding. According to CoBRA, bindings within the $\mathrm{VPPC}$ evolve more slowly over time through replay, which stands in contrast to MTL bindings that occur immediately during encoding. When $\mathrm{vPPC}$ bindings have been fully established, they have the capacity to reinstate detailed and rich ensembles of episodic features.
Given the presumed importance of vPPC to the establishment of long-lasting episodic memories, why do patients with PPC damage perform so well on tests of memory? It must be argued that intact MTL bindings in such patients mediate episodic retrieval, particularly if performance depends only on the retrieval of a small set of event features (e.g., old/new recognition memory). Consistent with CoBRA are findings of impairment in the ability to retrieve rich, context-bound episodic memories, such as recalling details of a past event and eliciting recollective (i.e., remember) or high-confident responses. Yet one might expect impaired source recognition in patients with PPC lesions, since such tests depend on memory for features associated with a specific context. It may be that source recognition, as typically assessed, requires only sparse multimodal bindings (e.g., associating a color to an object). It is predicted that patients with vPPC lesions should be impaired on source recognition tests that depend on retrieval of multiple or highly integrative source features. It must be further predicted that patients with vPPC lesions depend more on MTL bindings than do controls in retrieving episodic memories. Finally, particularly severe episodic retrieval deficits should occur in individuals with combined MTL and vPPC lesions. Of course patients with discrete, multiple lesions in these two regions would be rare. However, Alzheimer's disease does target these two regions, and it is clear that such patients exhibit severe and extensive impairment in the retrieval of remote autobiographical events (Kopelman, 1989).

CoBRA defines the role of the vPPC as establishing cortical bindings of event features. Thus, it is predicted that the successful retrieval of well-established semantic knowledge would not depend on vPPC bindings, since such retrievals are based on the activation of distributed semantic networks. Yet the retrieval of recently learned facts may depend on vPPC bindings to the extent that retrieval is facilitated by the reinstatement of episodic features associated with the fact. Recent findings support the role of the vPPC in the retrieval of recently learned facts, but not in the retrieval of well-learned (semantic) facts (Elman, Marian, Verstaen, \& Shimamura, 2011).

How can one account for vPPC activity in cognitive domains other than episodic retrieval? Findings suggest segregation of retrieval-related PPC activity and activity on tasks involving visual selective attention (Hutchinson et al., 2009; Sestieri et al., 2010). Less clear is the relationship between episodic retrieval processes and those involved in theory of mind, perspective taking, and future planning (Addis, Wong, \& Schacter, 2007; Saxe \& Kanwisher, 2003; Schacter, Addis, \& Buckner, 2007; Spreng and Grady 2009). All of these processes involve mentally displacing oneself to a different spatial-temporal context. Since CoBRA defines the vPPC as a means of integrating 
multimodal contextual features, the theory may be extended to suggest the importance of such bindings in all of these tasks. That is, as a convergence zone for spatial, object, and semantically based features, vPPC bindings may be critical any time one imagines oneself in a spatialtemporal context different from the one in which one is presently situated. Conversely, some vPPC regions may more specifically represent bindings of specific episodic events, whereas others are involved in imagining oneself in a different spatial-temporal context. Further cross-task analysis studies will likely define both commonalities and dissociations in regional activations across tasks involved in mentally placing oneself in the past, in the future, or in someone else.

What are the contributions of the $\mathrm{APPC}$ and $\mathrm{MPPC}$ ? The dPPC is presumed to be part of the dorsal path important for visuospatial processing and selective attention. It may also store spatial information-particularly, egocentric spatial properties. Thus, it is assumed that event features of a spatial nature are stored in the $\mathrm{APPC}$, since it is part of the dorsal visual path. The role of the mPPC is less clear. It is intricately related to visual processing and feeds information to the MTL. As such, the MPPC may be essential in transmitting information to the MTL. Retrosplenial lesions have been known to cause a severe amnesic disorder not unlike MTL amnesia (Valenstein et al., 1987). Alternatively, the mPPC may act as another convergence zone that links event features stored within or adjacent to these medial regions. The retrosplenial cortex has been implicated in emotionally laden episodic memory (Maddock, 1999), and thus the mPPC may be involved in linking emotional features of an episodic event to perceptual and conceptual features. On this view, mPPC may act as a convergence zone for integrating event features stored in medial regions, whereas the vPPC acts as a convergence zone for integrating event features stored in lateral regions.

\section{CoBRA theory in relation to other theories of episodic retrieval}

According to CoBRA, activity in vPPC regions during retrieval is indicative of successfully linking event features as a cortically bound ensemble. The degree or extent of vPPC activation should be correlated with the amount of information accumulated during retrieval. Thus, the predictions of CoBRA are, to some extent, consistent with M-ACC, although CoBRA assumes that vPPC activity is more involved than simply accumulating retrieved information, since it actually binds episodic memory representations. Recent findings by Guerin and Miller (2011) cast doubt on the $\mathrm{vPPC}$ as merely acting as an accumulator, since vPPC activity monitors overall memory strength, rather than the confidence of a response decision. With respect to E-BUFF, both it and CoBRA focus on the VPPC as linking retrieved information. Whereas E-BUFF considers this process as an independent working memory buffer that is dissociated from the actual representations that store event features (Baddeley, 2000), CoBRA considers vPPC regions as linking episodic features with long-term memory networks. In this way, CoBRA could be viewed as an activation model of E-BUFF, just as some have suggested that other working memory buffers are better viewed as activation of stored information, rather than as separate short-term memory systems (Anderson, 1983; Cowan, 1988).

According to AtoM, vPPC processes are closely associated with MTL bindings, since both involve the reinstatement of episodic features. According to CoBRA, vPPC bindings serve a very similar function as MTL bindings, since both act to bind episodic features. Thus, both AtoM and CoBRA suggest a close link between MTL and vPPC processes, although the functional processes defined by the two theories appear to be quite different. Whereas AtoM ties vPPC specifically to bottom-up processes, CoBRA considers its role as integrating relational activity specific to episodic events. To the extent that bottom-up processes refer to event features that are cortically bound by both MTL and VPPC, the two theories may have some direct similarities.

In summary, it is clear that episodic retrieval depends on a broad, integrative network of neural processes. No single component within this circuitry can be viewed as solely responsible for a recollective experience. At this moment in neurocognitive research, there is enough data to begin to develop functional characteristics of neural circuits involved in episodic encoding and retrieval, rather than pinning complex processes, such as recollection or consolidation, onto any single brain structure. CoBRA offers a straightforward, testable, and broad-based theory that integrates vPPC processes with well-established mechanisms, such as PFC executive control, MTL relational binding, and the representation of event features, such as visual features along the dorsal and ventral paths. To the extent that vPPC acts in the cortical binding of event features, it identifies a critical component in neurocognitive theories of memory, since it describes how episodic memories become well-integrated and durable neocortical representations.

\section{References}

Addis, D. R., Wong, A. T., \& Schacter, D. L. (2007). Remembering the past and imagining the future: Common and distinct neural substrates during event construction and elaboration. Neuropsychologia, 45, 1363-1377.

Aichhorn, M., Perner, J., Weiss, B., Kronbichler, M., Staffen, W., \& Ladurner, G. (2009). Temporo-parietal junction activity in 
theory-of-mind tasks: Falseness, beliefs, or attention. Journal of Cognitive Neuroscience, 21, 1179-1192.

Andersen, R. A., Asanuma, C., Essick, G., \& Siegel, R. M. (1990). Corticocortical connections of anatomically and physiologically defined subdivisions within the inferior parietal lobule. Journal of Comparative Neurology, 296, 65-113.

Andersen, R. A., \& Buneo, C. A. (2002). Intentional maps in posterior parietal cortex. Annual Review of Neuroscience, 25, 189-220.

Anderson, J. R. (1983). The architecture of cognition. Cambridge, MA: Harvard University Press.

Baddeley, A. (2000). The episodic buffer: A new component of working memory? Trends in Cognitive Sciences, 4, 417-423.

Badre, D. (2008). Cognitive control, hierarchy, and the rostro-caudal organization of the frontal lobes. Trends in Cognitive Sciences, 12, 193-200.

Baldo, J. V., \& Dronkers, N. F. (2006). The role of inferior parietal and inferior frontal cortex in working memory. Neuropsychology, 20, 529-538.

Bayley, P. J., Hopkins, R. O., \& Squire, L. R. (2003). Successful recollection of remote autobiographical memories by amnesic patients with medial temporal lobe lesions. Neuron, 38, 135-144.

Berryhill, M. E., Phuong, L., Picasso, L., Cabeza, R., \& Olson, I. R. (2007). Parietal lobe and episodic memory: Bilateral damage causes impaired free recall of autobiographical memory. Journal of Neuroscience, 27, 14415-14423.

Bruen, P. D., McGeown, W. J., Shanks, M. F., \& Venneri, A. (2008). Neuroanatomical correlates of neuropsychiatric symptoms in Alzheimer's disease. Brain, 131, 2455-2463.

Buccino, G., Binkofski, F., Fink, G. R., Fadiga, L., Fogassi, L., Gallese, V., et al. (2001). Action observation activates premotor and parietal areas in a somatotopic manner: an fMRI study. European Journal of Neuroscience, 13, 400-404.

Buckner, R. L., Andrews-Hanna, J. R., \& Schacter, D. L. (2008). The brain's default network anatomy, function, and relevance to disease. Annals of the New York Academy of Sciences, 1124, 1-38.

Buckner, R. L., \& Carroll, D. C. (2007). Self-projection and the brain. Trends in Cognitive Sciences, 11, 49-57.

Buckner, R. L., Koutstaal, W., Schacter, D. L., Dale, A. M., Rotte, M., \& Rosen, B. R. (1998). Functional-anatomic study of episodic retrieval: II. Selective averaging of event-related fMRI trials to test the retrieval success hypothesis. NeuroImage, 7, 163-175.

Budson, A. E., Sullivan, A. L., Mayer, E., Daffner, K. R., Black, P. M., \& Schacter, D. L. (2002). Suppression of false recognition in Alzheimer's disease and in patients with frontal lobe lesions. Brain, 125, 2750-2765.

Bunge, S. A. (2004). How we use rules to select actions: A review of evidence from cognitive neuroscience. Cognitive, Affective, \& Behavioral Neuroscience, 4, 564-579.

Cabeza, R., Ciaramelli, E., Olson, I. R., \& Moscovitch, M. (2008). The parietal cortex and episodic memory: An attentional account. Nature Reviews Neuroscience., 9, 613-625.

Cabeza, R., Dolcos, F., Graham, R., \& Nyberg, L. (2002). Similarities and differences in the neural correlates of episodic memory retrieval and working memory. NeuroImage, 16, 317-330.

Cabeza, R., \& St. Jacques, P. (2007). Functional neuroimaging of autobiographical memory. Trends in Cognitive Sciences, 11, 219 227

Cansino, S., Maquet, P., Dolan, R. J., \& Rugg, M. D. (2002). Brain activity underlying encoding and retrieval of source memory. Cerebral Cortex, 12, 1048-1056.

Cattaneo, L., \& Rizzolatti, G. (2009). The mirror neuron system. Archives of Neurology, 66, 557-560.

Cavada, C., \& Goldman-Rakic, P. S. (1989). Posterior parietal cortex in rhesus monkey: I. Parcellation of areas based on distinctive limbic and sensory corticocortical connections. Journal of Comparative Neurology, 287, 393-421.
Celsis, P., Boulanouar, K., Doyon, B., Ranjeva, J. P., Berry, I., Nespoulous, J. L., et al. (1999). Differential fMRI responses in the left posterior superior temporal gyrus and left supramarginal gyrus to habituation and change detection in syllables and tones. NeuroImage, 9, 135-144.

Christoff, K., \& Gabrieli, J. D. E. (2000). The frontopolar cortex and human cognition: Evidence for a rostrocaudal hierarchical organization within the human prefrontal cortex. Psychobiology, $28,168-186$.

Ciaramelli, E., Grady, C. L., \& Moscovitch, M. (2008). Top-down and bottom-up attention to memory: A hypothesis (AtoM) on the role of the posterior parietal cortex in memory retrieval. Neuropsychologia, 46, 1828-1851.

Clark, S. E., \& Gronlund, S. D. (1996). Global matching models of memory: How the models match the data. Psychonomic Bulletin \& Review, 3, 37-60.

Clower, D. M., West, R. A., Lynch, J. C., \& Strick, P. L. (2001). The inferior parietal lobule is the target of output from the superior colliculus, hippocampus, and cerebellum. Journal of Neuroscience, $21,6283-6291$.

Cohen, N. J., \& Eichenbaum, H. (1993). Memory, amnesia, and the hippocampal system. Cambridge, MA: MIT Press.

Corbetta, M., Kincade, J. M., Ollinger, J. M., McAvoy, M. P., \& Shulman, G. L. (2000). Voluntary orienting is dissociated from target detection in human posterior parietal cortex. Nature Neuroscience, 3, 292-297.

Corbetta, M., \& Shulman, G. L. (2002). Control of goal-directed and stimulus-driven attention in the brain. Nature Reviews Neuroscience, 3, 201-215.

Cowan, N. (1988). Evolving conceptions of memory storage, selective attention, and their mutual constraints within the human information-processing system. Psychological Bulletin, 104, 163-191.

Critchley, M. (1966). The parietal lobes. New York: Hafner.

Daselaar, S. M., Fleck, M. S., \& Cabeza, R. E. (2006). Triple dissociation in the medial temporal lobes: recollection, familiarity, and novelty. Journal of Neurophysiology, 96, 1902-1911.

Davidson, P. S., Anaki, D., Ciaramelli, E., Cohn, M., Kim, A. S., Murphy, K. J., et al. (2008). Does lateral parietal cortex support episodic memory? Evidence from focal lesion patients. Neuropsychologia, 46, 1743-1755.

D'Esposito, M. (2007). From cognitive to neural models of working memory. Philosophical Transactions of the Royal Society London $B, 362,761-772$.

de Zubicaray, G., McMahon, K., Eastburn, M., Pringle, A. J., Lorenz, L., \& Humphreys, M. S. (2007). Support for an auto-associative model of spoken cued recall: Evidence from fMRI. Neuropsychologia, 45, 824-835.

Dobbins, I. G., Foley, H., Schacter, D. L., \& Wagner, A. D. (2002). Executive control during episodic retrieval: Multiple prefrontal processes subserve source memory. Neuron, 35, 989-996.

Dobbins, I. G., \& Wagner, A. D. (2005). Domain-general and domainsensitive prefrontal mechanisms for recollecting events and detecting novelty. Cerebral Cortex, 15, 1768-1778.

Drowos, D. B., Berryhill, M., Andre, J. M., \& Olson, I. R. (2010). True memory, false memory, and subjective recollection deficits after focal parietal lobe lesions. Neuropsychology, 24, 265-275.

Egly, R., Driver, J., \& Rafal, R. D. (1994). Shifting visual attention between objects and locations: Evidence from normal and parietal lesion subjects, Journal of Experimental Psychology. General, 123, 161-177.

Eldridge, L. L., Engel, S. A., Zeineh, M. M., Bookheimer, S. Y., \& Knowlton, B. J. (2005). A dissociation of encoding and retrieval processes in the human hippocampus. Journal of Neuroscience, $25,3280-3286$ 
Elman, J. A., Marian, D. E., Verstaen, A., \& Shimamura, A. P. (2011). Ventral posterior parietal activation during retrieval of recently learned facts but not during retrieval of well-learned facts. San Francisco, CA: Paper presented at the Cognitive Neuroscience Society meeting.

Elman, J. A., \& Shimamura, A. P. (in press). Task relevance modulates successful retrieval effects during explicit and implicit memory tests. NeuroImage, 56, 345-353.

Gilboa, A., Ramirez, J., Kohler, S., Westmacott, R., Black, S. E., \& Moscovitch, M. (2005). Retrieval of autobiographical memory in Alzheimer's disease: relation to volumes of medial temporal lobe and other structures. Hippocampus, 15, 535-550.

Guerin, S. A., \& Miller, M. B. (2009). Lateralization of the parietal old/new effect: An event-related fMRI study comparing recognition memory for words and faces. NeuroImage, 44, 232-242.

Guerin, S. A., \& Miller, M. B. (2011). Parietal cortex tracks the amount of information retrieved even when it is not the basis of a memory decision. NeuroImage, 55, 801-807.

Haramati, S., Soroker, N., Dudai, Y., \& Levy, D. A. (2008). The posterior parietal cortex in recognition memory: Aneuropsychological study. Neuropsychologia, 46, 1756-1766.

Henson, R. N. A., Rugg, M. D., Shallice, T., Josephs, O., \& Dolan, R. J. (1999). Recollection and familiarity in recognition memory: An event-related functional magnetic resonance imaging study. Journal of Neuroscience, 19, 3962-3972.

Huijbers, W., Pennartz, C. M., \& Daselaar, S. M. (2010). Dissociating the "retrieval success" regions of the brain: Effects of retrieval delay. Neuropsychologia, 48, 491-497.

Hutchinson, J. B., Uncapher, M. R., \& Wagner, A. D. (2009). Posterior parietal cortex and episodic retrieval: Convergent and divergent effects of attention and memory. Learning and Memory, 16, 343-356.

Hwang, D. Y., Gallo, D. A., Ally, B. A., Black, P. M., Schacter, D. L., \& Budson, A. E. (2007). Diagnostic retrieval monitoring in patients with frontal lobe lesions: Further exploration of the distinctiveness heuristic. Neuropsychologia, 45, 2543-2552.

Janowsky, J. S., Shimamura, A. P., \& Squire, L. R. (1989). Source memory impairment in patients with frontal lobe lesions. Neuropsychologia, 27, 1043-1056.

Johnson, M. K., Hashtroudi, S., \& Lindsay, D. S. (1993). Source monitoring. Psychological Bulletin, 114, 3-28.

Kahn, I., Davachi, L., \& Wagner, A. D. (2004). Functionalneuroanatomic correlates of recollection: Implications for models of recognition memory. Journal of Neuroscience, 24, 4172-4180.

Kapur, S., Craik, F. I., Jones, C., Brown, G. M., Houle, S., \& Tulving, E. (1995). Functional role of the prefrontal cortex in retrieval of memories: A PET study. NeuroReport, 6, 1880-1884.

Klostermann, E. C., Kane, A. J. M., \& Shimamura, A. P. (2008). Parietal activation during retrieval of abstract and concrete auditory information. NeuroImage, 40, 896-901.

Klostermann, E. C., Loui, P., \& Shimamura, A. P. (2009). Activation of right parietal cortex during memory retrieval of nonlinguistic auditory stimuli. Cognitive, Affective,\&Behavioral Neuroscience, 9, 242-248.

Kopelman, M. (1989). Remote and autobiographical memory, temporal context memory and frontal atrophy in Korsakoff and Alzheimer patients. Neuropsychologia, 27, 437-460.

Leube, D. T., Erb, M., Grodd, W., Bartels, M., \& Kircher, T. T. (2003). Successful episodic memory retrieval of newly learned faces activates a left fronto-parietal network. Cognitive Brain Research, $18,97-101$.

Lynch, G., Rex, C. S., \& Gall, C. M. (2007). LTP consolidation: Substrates, explanatory power and functional significance. Neuropharmacology, 52, 12-23.

Maddock, R. J. (1999). The retrosplenial cortex and emotion: New insights from functional neuroimaging of the human brain. Trends in Neurosciences, 22, 310-316.
Majerus, S., Bastin, C., Poncelet, M., Van der Linden, M., Salmon, E., Collette, F., et al. (2007). Short-term memory and the left intraparietal sulcus: Focus of attention? Further evidence from a face short-term memory paradigm. NeuroImage, 35, 353-367.

Mangels, J. A., Gershberg, F. B., Shimamura, A. P., \& Knight, R. T. (1996). Impaired retrieval from remote memory in patients with frontal lobe damage. Neuropsychology, 10, 32-41.

Miller, E. K., \& Cohen, J. D. (2001). An integrative theory of prefrontal cortex function. Annual Review of Neuroscience, 24, 167-202.

Mitchell, K. J., \& Johnson, M. K. (2009). Source monitoring 15 years later: What have we learned from fMRI about the neural mechanisms of source memory? Psychological Bulletin, 135, 638-677.

Morris, R. G. M. (2006). Elements of a neurobiological theory of hippocampal function: The role of synaptic plasticity, synaptic tagging, and schemas. European Journal of Neuroscience, 23, 2829-2846.

Moscovitch, M., Rosenbaum, R. S., Addis, D. R., Westmacott, R., Grady, C., \& McAndrews, M. P., et al. (2003). Functional neuroanatomy of remote episodic, semantic, and spatial memory: A unified account based on multiple trace theory. Journal of Anatomy, 207, 35-66.

Moscovitch, M., Nadel, L., Winocur, G., Gilboa, A., \& Rosenbaum, R. S. (2006). The cognitive neuroscience of remote episodic, semantic and spatial memory. Current Opinion in Neurobiology, 16, 179-190.

Norman, K. A., \& O’Reilly, R. C. (2003). Modeling hippocampal and neocortical contributions to recognition memory: A complementarylearning-systems approach. Psychological Review, 110, 611-646.

Olson, I. R., \& Berryhill, M. (2009). Some surprising findings on the involvement of the parietal lobe in human memory. Neurobiology of Learning and Memory, 91, 155-165.

Paller, K. A., \& Wagner, A. D. (2002). Observing the transformation of experience into memory. Trends in Cognitive Sciences, 6, 92-102.

Pandya, D. N., \& Seltzer, B. (1982). Intrinsic connections and architectonics of posterior parietal cortex in rhesus monkeys. Journal of Comparative Neurology, 204, 196-210.

Paulesu, E., Frith, C. D., \& Frackowiak, R. S. (1993). The neural correlates of the verbal component of working memory. Nature, $362,342-345$.

Pause, M., Kunesch, E., Binkofski, F., \& Freund, H.-J. (1989). Sensorimotor disturbances in patients with lesions of the parietal cortex. Brain, 112, 1599-1625.

Rabin, J. S., Gilboa, A., Stuss, D. A., Mar, R. A., \& Rosenbaum, R. S. (2010). Common and unique neural correlates of autobiographical memory and theory of mind. Journal of Cognitive Neuroscience, 22, 1095-1111.

Ranganath, C., Johnson, M. K., \& D'Esposito, M. (2000). Left anterior prefrontal activation increases with demands to recall specific perceptual information. Journal of Neuroscience, 20, RC108.

Ranganath, C., Johnson, M. K., \& D'Esposito, M. (2003). Prefrontal activity associated with working memory and episodic long-term memory. Neuropsychologia, 41, 378-389.

Raposo, A., Han, S., \& Dobbins, I. G. (2009). Ventrolateral prefrontal cortex and self-initiated semanticelaboration during memory retrieval. Neuropsychologia, 47, 2261-2771.

Ratcliff, R. (1978). A theory of memory retrieval. Psychological Review, 85, 59-108.

Ratcliff, R., \& Starns, J. J. (2009). Modeling confidence and response time in recognition memory. Psychological Review, $116,59-83$.

Renier, L. A., Anurova, I., DeVolder, A. G., Carlson, S., VanMeter, J., \& Rauschecker, J. P. (2009). Multisensory integration of sounds and vibrotactile stimuli in processing streams for "what" and "where". Journal of Neuroscience, 29, 10950-10960. 
Rossi, S., Pasqualetti, P., Zito, G., Vecchio, F., Cappa, S. F., Miniussi, C., et al. (2006). Prefrontal and parietal cortex in human episodic memory: an interference study by repetitive transcranial magnetic stimulation. European Journal of Neuroscience, 23, 793-800.

Rounis, E., Yarrow, K., \& Rothwell, J. C. (2007). Effects of rTMS conditioning over the fronto-parietal network on motor versus visual attention. Journal of Cognitive Neuroscience, 19, 513-524.

Rugg, M. D., Fletcher, P. C., Chua, P. M., \& Dolan, R. J. (1999). The role of the prefrontal cortex in recognition memory and memory for source: An fMRI study. NeuroImage, 10, 520-529.

Rugg, M. D., Fletcher, P. C., Frith, C. D., Frackowiak, R. S., \& Dolan, R. J. (1996). Differential activation of the prefrontal cortex in successful and unsuccessful memory retrieval. Brain, 119, 20732083.

Rugg, M. D., \& Wilding, E. L. (2000). Retrieval processing and episodic memory. Trends in Cognitive Sciences, 4, 108-115.

Rushworth, M. F. S., Paus, T., \& Sipila, P. K. (2001). Attention systems and the organization of the human parietal cortex. Journal of Neuroscience, 21, 5262-5271.

Saletin, J. M., Goldstein, A. N., \& Walker, M. P. (in press). The role of sleep in directed forgetting of human memories. Cerebral Cortex.

Saxe, R., \& Kanwisher, N. (2003). People thinking about thinking people: The role of the temporo-parietal junction in "theory of mind. NeuroImage, 19, 1835-1842.

Schacter, D. L., Addis, D. R., \& Buckner, R. L. (2007). Remembering the past to imagine the future: The prospective brain. Nature Reviews Neuroscience, 8, 657-661.

Schacter, D. L., Curran, T., Galluccio, L., Milberg, W. P., \& Bates, J. F. (1966). False recognition and the right frontal lobe: A case study. Neuropsychologia, 34, 793-808.

Schacter, D. L., Verfaellie, M., \& Koutstaal, W. (2002). Memory illusions in amnesic patients: Findings and implications. In L. Squire \& D. L. Schacter (Eds.), Neuropsychology of memory (pp. 114-129). New York, NY: Guilford.

Serences, J. T., \& Yantis, S. (2006). Selective visual attention and perceptual coherence. Trends in Cognitive Sciences, 10, 38-45.

Serences, J. T., \& Yantis, S. (2007). Spatially selective representations of voluntary and stimulus-driven attentional priority in human occipital, parietal, and frontal cortex. Cerebral Cortex, 17, 284-293.

Sestieri, C., Shulman, G. L., \& Corbetta, M. (2010). Attention to memory and the environment: functional specialization and dynamic competition in human posterior parietal cortex. Journal of Neuroscience, 30, 8445-8456.

Shannon, B. J., \& Buckner, R. L. (2004). Functional-anatomic correlates of memory retrieval that suggest nontraditional processing roles for multiple distinct regions within the posterior parietal cortex. Journal of Neuroscience, 24, 10084-10092.

Shimamura, A. P. (2000). The role of the prefrontal cortex in dynamic filtering. Psychobiology, 28, 207-218.

Shimamura, A. P. (2002). Relational binding theory and the role of consolidation in memory retrieval. In L. R. Squire \& D. L. Schacter (Eds.), The neuropsychology of memory (pp. 61-72). New York: Guilford.

Shimamura, A. P. (2008). A neurocognitive approach to metacognitive monitoring and control. In J. Dunlosky \& R. A. Bjork (Eds.), Handbook of metamemory and memory (pp (pp. 373-390). New York: Psychology Press.

Shimamura, A. P. (2010). Hierarchical relational binding in the medial temporal lobe: The strong get stronger. Hippocampus, 20, 12061216.

Shimamura, A. P., \& Squire, L. R. (1991). The relationship between fact and source memory: Findings from amnesic patients and normal subjects. Psychobiology, 19, 1-10.
Shimamura, A. P., \& Wickens, T. D. (2009). Superadditive memory strength for item and source recognition: The role of hierarchical relational binding in the medial temporal lobe. Psychological Review, 116, 1-19.

Silver, M. A., \& Kastner, S. (2009). Topographic maps in human frontal and parietal cortex. Trends in Cognitive Sciences, 13, 488-495.

Simons, J. S., Peers, P. V., Hwang, D. Y., Ally, B. A., Fletcher, P. C., \& Budson, A. E. (2008). Is the parietal lobe necessary for recollection in humans? Neuropsychologia, 46, 1185-1191.

Simons, J. S., Peers, P. V., Mazuz, Y. S., Berryhill, M. E., \& Olson, I. R. (2010). Dissociation between memory accuracy and memory confidence following bilateral parietal lesions. Cerebral Cortex, 20, 479-485.

Simons, J. S., \& Spiers, H. J. (2003). Prefrontal and medial temporal lobe interactions in long-term memory. Nature Reviews Neuroscience, 4, 637-648.

Slotnick, S. D., \& Schacter, D. L. (2004). A sensory signature that distinguishes true from false memories. Nature Neuroscience, 7 , 664-672.

Spaniol, J., Davidson, P. S. R., Kim, A. S. N., Han, H., Moscovitch, M., \& Grady, C. L. (2009). Event-related fMRI studies of episodic encoding and retrieval: Meta-analysis using activation likelihood estimation. Neuropsychologia, 47, $1765-1779$.

Spreng, R. N., \& Grady, C. L. (2009). Patterns of brain activity supporting autobiographical memory, prospection, and theory of mind, and their relationship to the default mode network. Journal of Cognitive Neuroscience, 22, 1112-1123.

Squire, L. R., Haist, F., \& Shimamura, A. P. (1989). The neurology of memory: Quantitative assessment of amnesia in two groups of amnesic patients. Journal of Neuroscience, 9, 828-839.

Squire, L. R., Shimamura, A. P., \& Amaral, D. G. (1989). Memory and the hippocampus. In J. H. Byrne \& W. O. Berry (Eds.), Neural models of plasticity: Experimental and theoretical approaches (pp. 208-239). San Diego, CA: Academic Press.

Squire, L. R., \& Zola, S. M. (1998). Episodic memory, semantic memory, and amnesia. Hippocampus, 8, 205-211.

Svoboda, E., McKinnon, M. C., \& Levine, B. (2006). The functional neuroanatomy of autobiographical memory: A meta-analysis. Neuropsychologia, 44, 2189-2208.

Tulving, E. (2002). Episodic memory: from mind to brain. Annual Review of Psychology, 53, 1-25.

Tulving, E., Kapur, S., Markowitsch, H. J., Craik, F. I., Habib, R., \& Houle, S. (1994). Neuro-anatomical correlates of retrieval in episodic memory: auditory sentence recognition. Proceedings of the National Academy of Sciences, 91, 2012-2015.

Uncapher, M. R., \& Wagner, A. D. (2009). Posterior parietal cortex and episodic encoding: Insights fromfMRI subsequent memory effects and dual-attention theory. Neurobiology of Learning and Memory, 91, 139-154.

Valenstein, E., Bowers, D., Verfaellie, M., Heilman, K. M., Day, A., \& Watson, R. T. (1987). Retrosplenial amnesia. Brain, 110, 16311646.

Vilberg, K. L., \& Rugg, M. D. (2008). Memory retrieval and the parietal cortex: A review of evidence from a dual-process perspective. Neuropsychologia, 46, 1787-1799.

Vincent, J. L., Snyder, A. Z., Fox, M. D., Shannon, B. J., Andrews, J. R., Raichle, M. E., et al. (2006). Coherent spontaneous activity identifies a hippocampal-parietal mnemonic network. Journal ofNeurophysiology, 96, 3517-3531.

Wagner, A. D. (2002). Cognitive control and episodic memory: Contributions from prefrontal cortex. In L. R. Squire \& D. L. Schacter (Eds.), Neuropsychology of memory (3rd ed., pp. 174192). New York: Guilford. 
Wagner, A. D., Shannon, B. J., Kahn, I., \& Buckner, R. L. (2005). Parietal lobe contributions to episodic memory retrieval. Trends in Cognitive Sciences, 9, 445-453.

Warrington, E. K., \& Shallice, T. (1969). The selective impairment of auditory verbal short-term memory. Brain, 92, 885-896.

Wheeler, M. E., \& Buckner, R. L. (2003). Functional dissociation among components of remembering: Control, perceived oldness, and content. Journal of Neuroscience., 23, 3869-3880.

Wheeler, M. E., \& Buckner, R. L. (2004). Functional-anatomic correlates of remembering and knowing. Neurolmage, 21, 1337-1349.

Yantis, S., Schwarzbach, J., Serences, J. T., Carlson, R. L., Steinmetz, M. A., Pekar, J. J., et al. (2002). Transient neural activity in human parietal cortex during spatial attention shifts. Nature Neuroscience, 5, 995-1002.

Yonelinas, A. P. (2002). The nature of recollection and familiarity: A review of 30 years of research. Journal of Memory and Language, 46, 441-517.

Yonelinas, A. P., Otten, L. J., Shaw, K. N., \& Rugg, M. D. (2005). Separating the brain regions involved in recollection and familiarity in recognition memory. Journal of Neuroscience., $25,3002-3008$.

Young, L., \& Saxe, R. (2008). The neural basis of belief encoding and integration in moral judgment. NeuroImage, 40, 1912-1920. 